\title{
SPORESAT: A NANOSATELLITE PLATFORM LAB-ON-A-CHIP SYSTEM FOR INVESTIGATING GRAVITY THRESHOLD OF FERN-SPORE SINGLE-CELL CALCIUM ION CURRENTS
}

\author{
W.W.A. Wan Salim ${ }^{*}$, J. Park ${ }^{1}$, J.L. Rickus ${ }^{1}$, A. Rademacher ${ }^{2}$, A.J. Ricco ${ }^{2}$, A. Schooley ${ }^{2}$, J. Benton ${ }^{2}$, B. Wickizer ${ }^{2}$, \\ A. Martinez ${ }^{2}$, N. Mai', M. Rasay ${ }^{3}$, D.M. Porterfield ${ }^{1}$, L. Brownston ${ }^{2}$, M. Cote ${ }^{2}$, G. Defouw ${ }^{2}$, M. Henschke ${ }^{2}$, \\ C. Kitts ${ }^{3}$, E. Luzzi ${ }^{2}$, M. Perez ${ }^{2}$, S. Roux ${ }^{4}$, M. Salmi ${ }^{4}$, and A. Sweet ${ }^{2}$ \\ ${ }^{1}$ Purdue University, West Lafayette, IN, USA \\ ${ }^{2}$ NASA Ames Research Center, Moffett Field, CA, USA \\ ${ }^{3}$ Santa Clara University, Santa Clara, CA, USA \\ ${ }^{4}$ University of Texas, Austin, TX, USA
}

\begin{abstract}
SporeSat - a lab-on-a-chip (LOC) centrifuge platform designed for integration as the payload of a small $(5.5 \mathrm{~kg})$, freeflying satellite - has been developed to determine the gravitational thresholds for calcium-ion channel activation of a single-cell spore from the fern Ceratopteris richardii. This fern is an important model system for gravity-directed plant-cell development during variable-gravity conditions attainable only in space flight. Calcium-ion channel activity is measured by photolithographically defined calcium ion-selective electrodes (ISEs) at opposite ends of each spore. Artificial gravity is created by rotating a disk-like platform that contains the spores in wells along with the calcium ISEs. Ground experiments reveal a maximum calcium concentration ratio at $2.2 \times g$, between micro-ion-selective electrodes near the "top" and "bottom" ends of the spore, indicating an increasing calcium concentration at one "end" of the fern spore with respect to the other. Confocal micrographs of rhizoid formation confirm the light-induced germination. SporeSat is a spaceflight experiment that will take $\sim 4$ days; data will be telemetered to Earth over 100 days.
\end{abstract}

\section{INTRODUCTION}

Plants and plant cells are known to have sensors for external cues, but the molecular mechanism by which they sense gravity remains to be determined. Plants have no ambulatory function, but possess a basic capability to sense the intensity of the $g$-force [1]. To understand plant gravisensing, researchers have utilized spores of the fern Ceratopteris richardii as a gravitational plant model system, because the spore is unicellular, has a short life span, and is easily handled and cultured [2].

Previous studies using self-referencing ion-selective (SERIS) electrodes and cell-electrophysiology-lab-on-a-chip (CEL-C) technologies have found that calcium ions play a role in gravitydirected polarity development of the $C$. richardii fern spore. Calcium channel and pump activity appear to be influenced by gravity [3]. Polarity of calcium transport was observed in ground studies with a SERIS electrode: a strong efflux of $\mathrm{Ca}^{2+}$ at the top and a strong influx at the bottom of the spore. This polar ion flux was found to occur at 6-12 hours from the onset of germination initiated by light. However, studies on fern spores utilizing the SERIS electrode lack temporal resolution. The CEL-C biochip developed later has the desired temporal resolution while serving as a template for simultaneous measurements of $\mathrm{Ca}^{2+}$ concentration on 16 spores [4]. However, the CEL-C is stationary. When flown on parabolic flights the CEL-C was used to observe the effect of micro- $g$ and hyper- $g$ levels on spore calcium flux. In these experiments, the magnitude of the $\mathrm{Ca}^{2+}$ ratio between the two "ends" of a spore was highest at $2 \mathrm{x} g$, and this concentration ratio decreased at $0.01 \times g$. There was a lag of 10-15 seconds before a change in $\mathrm{Ca}^{2+}$ concentration was observed in the fern spores when going from hyper- $g$ to micro- $g$, whereas the lag was only 2 seconds from micro- $g$ to hyper- $g$ [5]. From these initial results, it was hypothesized that calcium-ion channels and pumps are activated at gravity levels in between these two extreme $g$-values.

To determine the onset of the calcium-channel and pump activation that produces the $\mathrm{Ca}^{2+}$ current polarity, we are developing a cell-electrophysiology-lab-on-a-chip (CEL-C) advanced bioCD, or in short, the bioCD. The bioCD aims to investigate the gravitational threshold for calcium-ion channel and pump activation in the single-cell spore of the fern Ceratopteris richardii. The bioCD enables finer investigation of $C$-richardii gravisensing; it has a rotating centrifuge format that provides varying artificial gravity levels in microgravity, with capability for simultaneous $\mathrm{Ca}^{2+}$ current measurements.

\section{SPORESAT PAYLOAD \\ Payload Design}

SporeSat [6] is a nanosatellite $(10 \times 10 \times 34 \mathrm{~cm}, 5.5 \mathrm{~kg})$, the payload of which integrates motors, thermal control, measurement electronics, and various sensors with three bioCDs, all housed in a container with an internal pressure of $1 \mathrm{~atm}$ (Fig. 1). Two bioCDs rotate; the third remains stationary as a control. A bioCD, fabricated on 2-inch fused-silica substrate (Fig. 2), includes thirtytwo $126-\mu \mathrm{m}$-diameter cylindrical SU-8 wells (1:1 aspect ratio), each housing one fern spore. Four $\mathrm{Pt}$ resistance-temperature devices (RTDs) per bioCD provide closed-loop thermal control. A pair of ion-selective electrodes (ISEs) measures the ratio of $\mathrm{Ca}^{2+}$ concentrations at either "end" of each fern spore. When the bioCD rotates, spores are subject to different gravitation magnitudes based on their distance from the rotational axis ( 8 wells at each of 4 distances) and rotational velocity (Table 1).

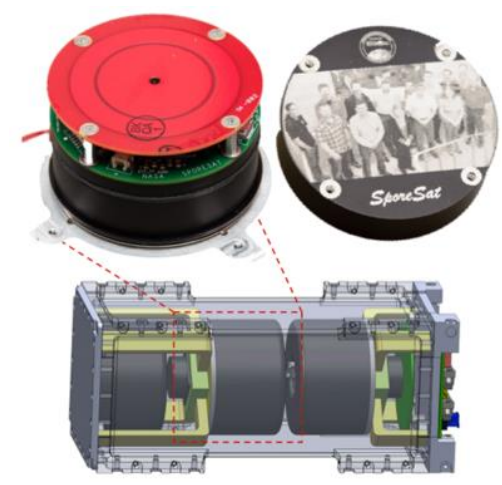

Figure 1: SporeSat payload showing location of 3 bioCD assemblies inside a 1-atm container. Each bioCD rotating assembly measures differential calcium-ion concentrations from germinating fern spores. 


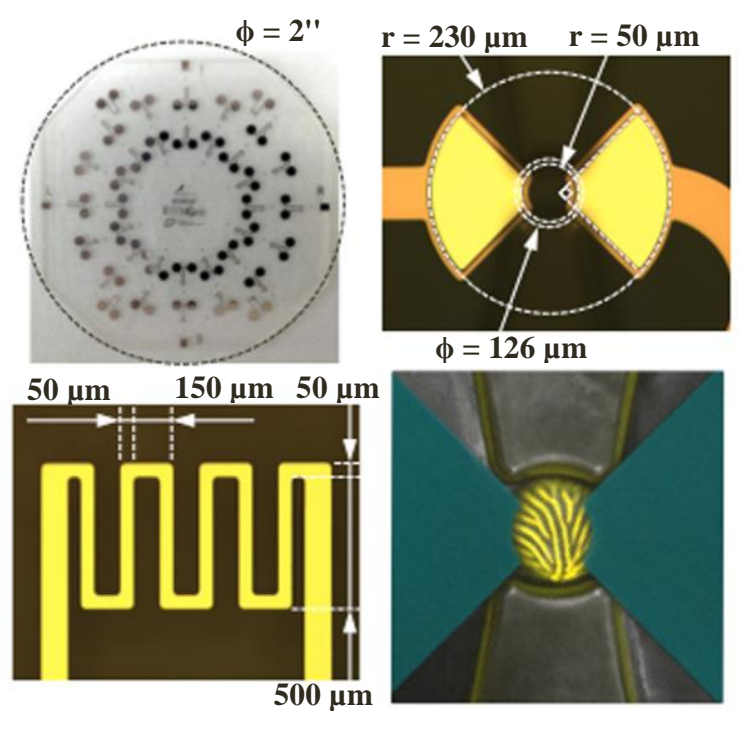

Figure 2: BioCD design showing 32 wells on a 2-inch-diameter area (photo, top left). Eight wells are located at each of four radial distances from the rotational axis. Each well has a pair of wedge-shaped ISEs (top right) on either side of a single fern spore (bottom right, held between SU-8 "pincers"). Four thin-film RTDs (bottom left) are located near the outer wells.

Table 1: Artificial gravity generated from rotation at wells located at various radial distances. The $\mathrm{g}$ levels vary from $2 \mathrm{xg}$ to $0.06 \mathrm{xg}$.

\begin{tabular}{|c|c|c|c|c|c|}
\hline \multicolumn{2}{|c|}{ Rotation rate } & \multicolumn{4}{|c|}{ Gravity at $\mathbf{R}(\mathbf{m m})$} \\
\hline (rpm) & $\mathbf{( H z )}$ & $\mathbf{R}_{\mathbf{1}}=\mathbf{9}$ & $\mathbf{R}_{\mathbf{2}}=\mathbf{1 2}$ & $\mathbf{R}_{\mathbf{3}}=\mathbf{1 5}$ & $\mathbf{R}_{\mathbf{4}}=\mathbf{1 8}$ \\
\hline 80 & 1.38 & $0.06 \times \mathrm{x} g$ & $0.09 \times \mathrm{x} g$ & $0.11 \times g$ & $0.13 \times g$ \\
\hline 112 & 1.87 & $0.13 \times \mathrm{x}$ & $0.17 \times \mathrm{x}$ & $0.21 \times g$ & $0.25 \times g$ \\
\hline 158 & 2.63 & $0.25 \times \mathrm{x} g$ & $0.34 \times g$ & $0.42 \times g$ & $0.50 \times g$ \\
\hline 223 & 3.72 & $0.50 \times \mathrm{x}$ & $0.67 \times \mathrm{x} g$ & $0.83 \times g$ & $1.00 \times g$ \\
\hline 315 & 5.25 & $1.00 \times \mathrm{x} g$ & $1.33 \times g$ & $1.66 \times g$ & $2.00 \times g$ \\
\hline
\end{tabular}

\section{bioCD Fabrication and Spore Localization}

The bioCD is fabricated via a four-mask process. Mask one is for electrical connection and RTD definition, mask two is for exposing the conductive active electrode by etching away the oxide passivation layer, mask three is for $\mathrm{Ag}$ deposition on active electrode areas, and mask four is for SU-8 spore-well fabrication. Afterwards, the bioCD Ag electrodes are chlorided in sodium hypochlorite $(\mathrm{NaClO})$, resulting in $\mathrm{Ag} / \mathrm{AgCl}$ electrodes, and subsequently spin-coated with an ion-selective membrane (ISM) specific for $\mathrm{Ca}^{2+}$. The Pt-Ag/AgCl-ISM layer is called an ionselective electrode (ISE). Figure 3 shows the fabricated crosssectional schematic at the final stage of each mask process, and the surface functionalization of the electrodes.

Each ISE operates based on the potentiometric electrochemical principle. The ISE reacts with $\mathrm{Ca}^{2+}$ and outputs an electrical signal that is proportional to the $\mathrm{Ca}^{2+}$ concentration. The Nernst equation defines the concentration of $\mathrm{Ca}^{2+}$ at equilibrium; the potential difference, which exists at both "ends" of a spore, is translated into a voltage value. A plot of measured potential vs $\mathrm{Ca}^{2+}$ concentration is linear if the ISM is perfectly selective. Calibration of the ISE for $\mathrm{Ca}^{2+}$ reveals an average Nernstian slope of $\sim 30 \mathrm{mV} /$ decade change in $\mathrm{Ca}^{2+}$ concentration.
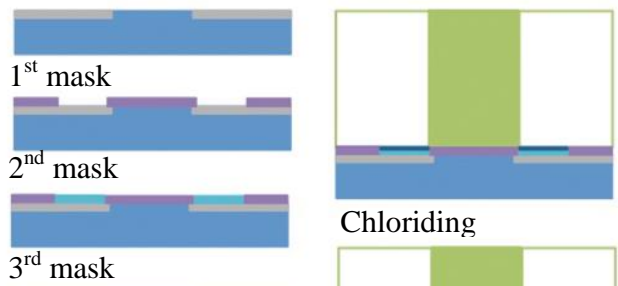

Chloriding
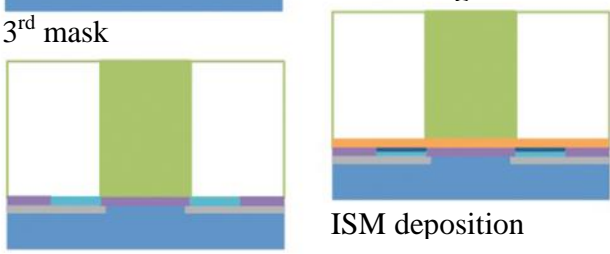

$4^{\text {th }}$ mask

ISM deposition

$=$ Fused silica $\approx \mathrm{SiO}_{2} \approx \mathrm{SU}-8=\mathrm{Pt} \quad \approx \mathrm{Ag}=\mathrm{Ag} / \mathrm{AgCl} \equiv \mathrm{ISM}$

Figure 3: bioCD electrode fabrication and surfacefunctionalization process cross-sectional schematic.

After $\mathrm{Ag} / \mathrm{AgCl}$ electrode preparation and ion-selective membrane deposition, bioCDs are conditioned for $\sim 24 \mathrm{hr}(1 \mu \mathrm{M}$ $\mathrm{CaCl}_{2} / 10 \mu \mathrm{M} \mathrm{KNO}_{3}$ ) before loading one spore (126 $\mu \mathrm{m}$ diameter) per well. Figure 4 shows the cross-sectional schematic of a spore in a well.

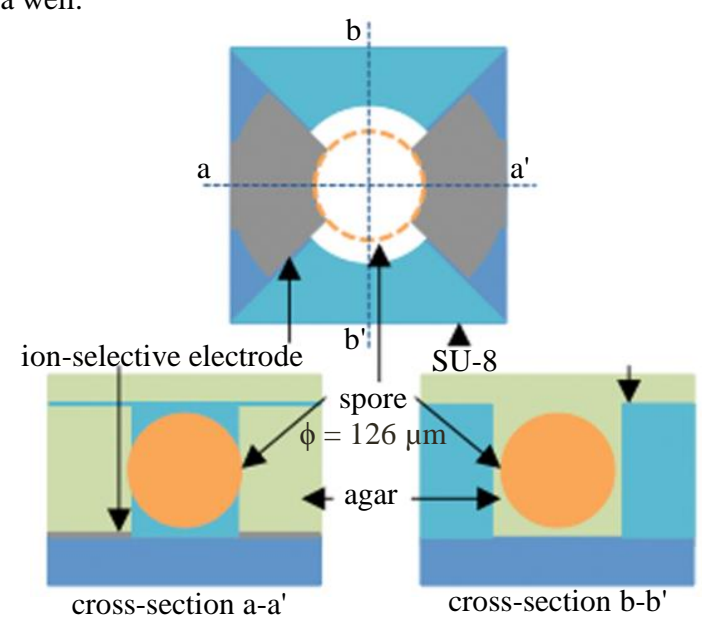

Figure 4: Cross-sectional schematic of a spore in a bioCD well. The SU-8 epoxy features along $b$ - $b$ ' form a "pincer" that, along with agar gel, holds the spore in place. ISEs are located beneath the "ends" of the spore closest to and furthest from the center of rotation.

Each bioCD is housed in a sealed assembly (Fig. 5) containing two layers of agar gel $-0.5 \%$ to provide high ionic mobility in a hydrated environment between two ISEs, and $2.0 \%$ to immobilize the spore and preventing it from dislodging during shock and vibration. Integral co-rotating circuit boards with local batteries, contacting each bioCD via pogo-pin arrays, include 32 differential amplifiers to measure and digitize $\mathrm{Ca}^{2+}$ concentration ratios, as well as controlling a lighting system (red OLED disk) that germinates the spores through the bottom of the transparent bioCD. Digital signals are transmitted capacitively across a sub$\mathrm{mm}$ gap to a mating stationary PC board. 


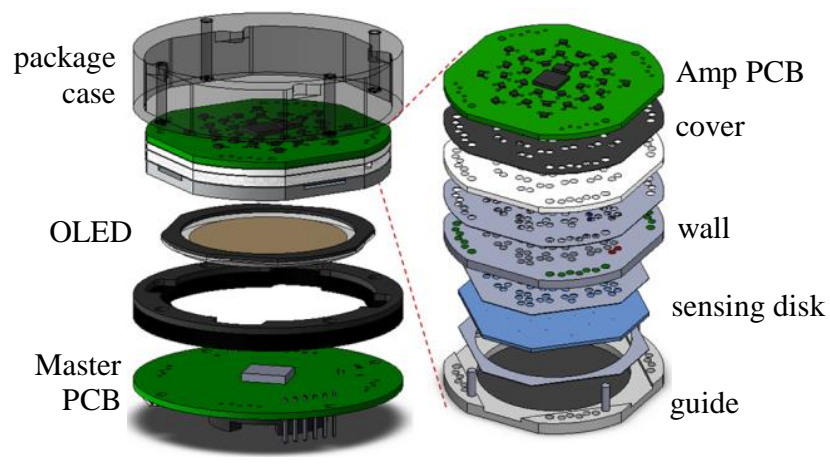

Figure 5: Exploded-view schematic of the bioCD rotating assembly. The bioCD package consists of the enclosed assembly (EA) unit, which seals the agar that surrounds the spores in the wells to prevent drying out and maintain spore viability for as long as 90 days.

\section{GROUND EXPERIMENTS AND DISCUSSION}

Experiments were conducted on the ground to test the system. Fern spores were sterilized in $20 \%$ Clorox ${ }^{\circledR}$ bleach, and loaded into the bioCD in the dark under green safety light; the bioCD was then sealed into the container and stored to simulate prespaceflight stasis during launch-vehicle integration. Zero-day tests were conducted: spores were exposed to 2 hours of red light at 620 $\mathrm{nm}$ peak wavelength and rotation at $290 \mathrm{rpm}$ (simulated gravity levels at $1.1,1.5,1.8$, and $2.2 \mathrm{xg}$ from inner to outer radii).

In these tests, spores were stored for 3 weeks and five days in agar medium before exposure to light and rotation. Furthermore, the bioCD also contains negative controls; these are wells filled with agar or a glass bead at all $g$-levels. Figure 6 shows the calcium concentration ratio of representative ISE pairs for fern spores at 2.2, 1.8, 1.5, and 1.1xg, a glass bead, and an empty agar well. A higher differential ratio indicates increasing $\left[\mathrm{Ca}^{2+}\right]_{\text {outer }} /\left[\mathrm{Ca}^{2+}\right]_{\text {inner }}$ on either "end" of the fern spore. A higher differential ratio implies larger $\left[\mathrm{Ca}^{2+}\right]$ differences between the two "ends" of the fern spore.

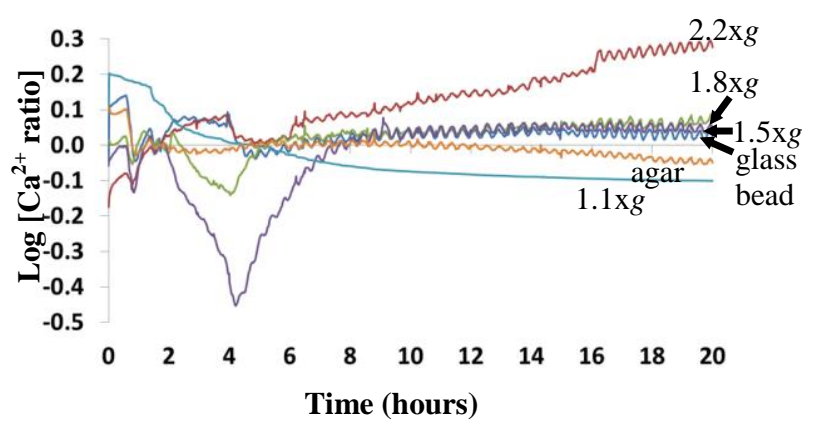

Figure 6: Calcium concentration differential measured during ground experiments for an ISE pair at either "end" of a germinating fern spore under rotation $(f=290 \mathrm{rpm})$ after a $2-\mathrm{hr}$ red-light exposure, for wells experiencing 2.2, 1.8, 1.5, and 1.1xg, in comparison to wells that contain empty agar or agar with a glass bead.

From Figure 6, we can state the following: the magnitude of the $\left[\mathrm{Ca}^{2+}\right]$ ratio is highest at $2.2 \mathrm{xg}$; the $\left[\mathrm{Ca}^{2+}\right]$ ratio of fern spores at the other $g$-levels does not significantly differ from that of the glass bead and empty agar. From these results, we can say that the signals from germinating spores at the highest $g$-level differ significantly from those from an empty well or a glass bead. It is important to note that ground studies do not emulate what the spores will experience at microgravity since the spore is being subject to the vector sum of both Earth and centrifugal gravities, where the resultant force vector is not well aligned with the axis of the two ISEs. Since the $\left[\mathrm{Ca}^{2+}\right]$ ratio is between the two ends of a spore, one end having an efflux of $\left[\mathrm{Ca}^{2+}\right]$ and the other end having an influx of $[\mathrm{Ca} 2+]$, the ISE location would be at the wrong place to measure the efflux, especially for the lower $g$ levels. At the highest $g$-level, the vector sum shifts to being more at the plane of the bioCD, which will bring one "top " of a spore closer to one of the ISEs.

To understand electrical signals obtained from the system, we conducted confocal imaging immediately after the zero-day tests, to visualize spore physical development. Spore development begins with the cracking of a trilete marking on the spore coat, followed by emergence of the primary rhizoid from this opening in the spore coat. In these studies, spore germination is defined as visible rhizoid emergence from the spore. Figure 7 shows a confocal micrograph of a germinated spore in a zero-day test bioCD after rhizoid formation. The germinated fern spores in the bioCD confirm that the lighting and environmental conditions are sufficient for spores to germinate.

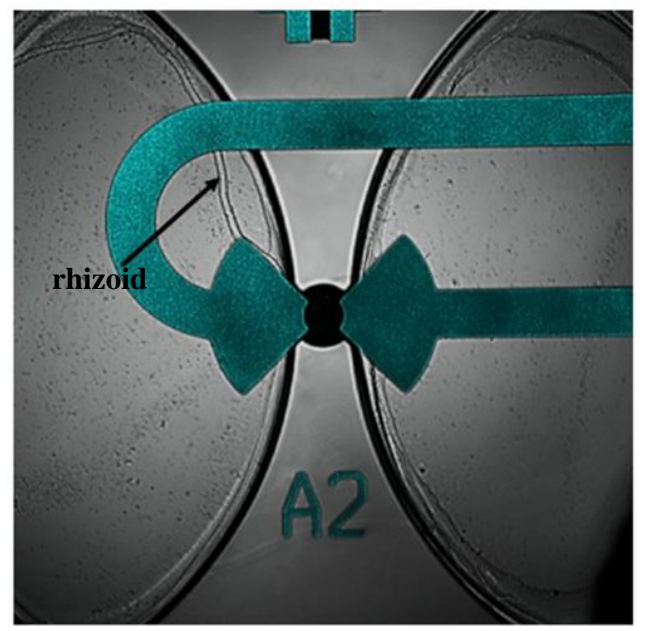

Figure 7: Representative confocal micrograph image of a spore with primary rhizoid in a well.

Previous studies using CEL-C have confirmed that the $\mathrm{Ca}^{2+}$ current polarity in a single-cell fern spore is directed by gravity. SporeSat provides a variety of artificial gravities to determine the gravity threshold of polarity development. SporeSat inherits the dual-electrode-differential-coupling (DEDC) measurement method of the CEL-C [4], where the $\mathrm{Ca}^{2+}$ concentration ratio between two "ends" of a spore is measured instead of absolute concentration. In DEDC, two electrodes reference each other; perfectly matched electrodes will give a voltage reading of $0 \mathrm{~V}$. Subtle changes in one electrode will give a large signal amplification.

CEL-C differs from SporeSat in overall biochip design, most significantly in the electrode geometry. The CEL-C had an active electrode area of $1660 \mu \mathrm{m}^{2}$; one electrode was located on each of the four sides of an etched pyramidal silicon well $\left(150 \mu \mathrm{m}^{2}\right)$, with a spore located inside the well. All poles of a spore were within $\sim 10 \mu \mathrm{m}$ of the electrodes. The bioCD wedge-shaped active electrode area is $35909 \mu \mathrm{m}^{2}$, but only $10537 \mu \mathrm{m}^{2}$ touch the spore at 
each "end." The spore is contained in an etched SU-8 well. The distance of an ISE to a spore for both systems is limited to less than $10 \mu \mathrm{m}$ in order to minimize the diffusion distance of the $\mathrm{Ca}^{2+}$ ions to the ISE and to prevent signal loss.

Conditions relating to the spore also differ between the two systems in terms of lighting for germination. The CEL-C system had no integrated lighting system, spores being exposed to ambient light continuously before and during measurements, whereas the bioCD system is kept in the dark and spores are simply exposed to red light (peak $\lambda=620 \mathrm{~nm}$ ) for 2 hours to initiate germination. Thanks to the transparent bioCD substrate, uniform lighting and time of exposure to the spores are attainable. The CEL-C could not achieve this because of its opaque substrate.

Owing to the differences in electrode geometry and lighting system between the CEL-C and SporeSat systems, results are not parallel. It can be expected that the $\left[\mathrm{Ca}^{2+}\right]$ ratio is less in the SporeSat owing to the smaller electrode area touching the spore and the larger total electrode area. Furthermore, spores experience only 2 hours of lighting in the bioCD, in comparison to the continuous lighting in the CEL-C.

Although these are not parallel systems, both the CEL-C and the SporeSat are viable tools to study fern-spore electrophysiology: they utilize a similar electrochemical measurement method; both systems measure the differential voltage that are translated to a ratio of $\mathrm{Ca}^{2+}$ concentration on both sides of the spore. A 24-hour germination experiment on the ground can be correlated with previous SERIS experiments, but with tremendously enhanced throughput.

\section{CONCLUSIONS}

Previous CEL-C experiments in microgravity reveal that gravity is locally affecting transcellular $\mathrm{Ca}^{2+}$ with mechanosensory $\mathrm{Ca}^{2+}$ ion channels as the major player in the mechanism. The transcellular $\mathrm{Ca}^{2+}$ flux closely follows the rapidly changing gravity force, with hyper- $g$ polarizing the current while micro- $g$ results in a zero magnitude of calcium current. However, what we still do not know is the gravity threshold of fern-spore polarity development from $\mathrm{Ca}^{2+}$ ions. The SporeSat system aims to provide the answer, by providing several artificial gravity levels to the spore in microgravity. Ground studies with the bioCD reveal a significant $\left[\mathrm{Ca}^{2+}\right]$ ratio for fern spores at $2.2 \times g$ in comparison to glass bead and agar-only wells, while at lower $g$-levels the ratios trend more closely to the glass bead and empty wells. Confocal imaging has proved that spores do germinate (formation of rhizoid) in the bioCD wells under the lighting and storage conditions, but more studies are needed to find the optimal lighting condition for the spores.
The platforms can easily be tailored to fit other cell types by changing the well or electrode design, making the CEL-C and SporeSat systems especially suitable for single-cell electrophysiological measurements. From a microfabrication standpoint, changing well size and electrode geometry is simply changing the masks that are used to pattern the features on the substrate. Furthermore, to modify the applications of the system, one can always change the surface functionalization process.

Under the requirements of spaceflight research, such as payload constraints and limited crew time, electrochemical sensor use is especially attractive. Furthermore, electrochemical sensors are also easily packaged and integrated with spaceflight hardware systems, allowing for sensor operation to be made autonomous. The SporeSat will be the first electrochemical sensor system in a LOC format whose functionality will be demonstrated in Space.

\section{REFERENCES}

[1] A.H. Brown, "The Influence of Gravity on the Structure and Functions of Plant Material", Advances in Space Research, 4, 12 (1984).

[2] A. Chatterjee, and S. J. Roux, "Ceratopteris richardii: A Productive Model for Revealing Secrets of Signaling and Development", Journal of Plant Growth Regulation, 19, 3 (2000).

[3] A. Chatterjee, D. M. Porterfield, P.S. Smith, and S. J. Roux, "Gravity-directed Calcium Current in Germinating Spores of Ceratopteris richardii," Planta, 210, 4 (2000).

[4] A. Ul Haque, M. Rokkam, A. R. Decarlo, S. T. Wereley, S. J. Roux, P. P. Irazoqui, and D. M. Porterfield, "A MEMS Fabricated Cell Electrophysiology Biochip for In Silico Calcium Measurements", Sensors and Actuators B: Chemical, 123, 1 (2007).

[5] M. L. Salmi, A. Ul Haque, T. J. Bushart, S. C. Stout, S. J. Roux, and D. M. Porterfield, "Changes in Gravity Rapidly Alter the Magnitude and Direction of a Cellular Calcium Current", Planta 233, 5 (2011).

[6] The SporeSat Mission is a Mission of Opportunity ("MoO-2") funded by the NASA Human Exploration and Operations Mission Directorate (HEOMD).

\section{CONTACT}

*W.W.A. Wan Salim, amanisalim6@gmail.com 